\title{
Stroke rehabilitation and research: consideration of the role of the cortico-reticulospinal system
}

\author{
Augusta Silva ${ }^{a}$, Julie Vaughan-Graham ${ }^{b}$, Claudia Silva $^{c}$, Andreia Sousa $^{c}$, Christine Cunha $^{c, d}$, \\ Rosália Ferreira ${ }^{c, d}$ and Pedro Maciel Barbosa ${ }^{c, e}$ \\ aPhysiotherapy Department, School of Health, Polytechnic Institute of Porto, Center for Rehabilitation Research - Center of Human \\ Movement and Human Activity, Porto, Portugal; ${ }^{b}$ Department of Physical Therapy, University of Toronto, Toronto, Canada; ${ }^{C}$ Physiotherapy \\ Department, School of Health, Polytechnic Institute of Porto, Center for Rehabilitation Research - Center of Human Movement and Human \\ Activity, Porto, Portugal; ${ }^{\mathrm{d}}$ Sport Faculty - University of Porto, Porto, Portugal; ${ }^{\mathrm{e}}$ Institute of Public Health - University of Porto, \\ Porto, Portugal
}

\begin{abstract}
A cerebrovascular accident, otherwise known as stroke, has the potential to damage multiple areas within the brain affecting descending motor control via a multitude of pathways resulting in a wide variety of movement problems. The cortico-reticulospinal system, one of the largest motor systems, is frequently affected, compromising its output, resulting in postural control deficits. The identification of clinically relevant instruments and scales to document and evaluate recovery in post-stroke patients is vital. However, the availability of such measures and scales which take into consideration the role of postural control as an integral component of functional movement performance are scarce. This paper will critically discuss the importance of integrating current neuroscience and motor control knowledge in order to better understand and describe the clinical presentation of persons post-stroke such that the effectiveness of stroke rehabilitation can be appropriately measured.
\end{abstract}

\section{KEYWORDS}

Stroke; measures; corticoreticulospinal system

\section{Introduction}

Impaired motor control, with a subsequent loss in functional capability, is one of the primary consequences of stroke (Dimyan and Cohen 2011; Grefkes and Ward 2014). Ischemic events, involving the middle cerebral artery (MCA), are the most common type of stroke (de Niet et al. 2007; Mohr et al. 2011), frequently compromising both cortical and subcortical connectivity and output from cortical regions such as the pre-motor, primary and supplementary motor areas (Lemon 2008; Grefkes et al. 2010). It is now understood that stroke events rarely result in a pure cortico-spinal lesion as cortico-reticular axons, which significantly outnumber cortico-spinal axons, are also simultaneously affected (Lemon 2008; Rothwell 2009; Baker 2011).

Cortico-reticulospinal damage results in bilateral postural control impairments, as well as movement impairments of the contra-lesional side (Prentice and Drew 2001; Rothwell 2012), due to the predominantly ipsilateral (pontine) and bilateral (medullary) distributions of reticulospinal pathways (Takakusaki et al. 2016). This knowledge assumes particular relevance considering that movement performance requires an appropriate postural orientation of all segments of the body in relation to the environment and gravity while keeping an upright alignment for stability (Horak 2006; Bouisset and Do 2008; Guigon 2010; Latash and Zatsiorsky 2016; Latash 2016; Barra et al. 2017). Movement is generated by the activity of neuronal circuits collecting and integrating information, resulting in precisely timed skeletal muscle contractions (Arber 2012). This dynamic and complex interplay between neurological and muscular systems enables effective motor control (Latash 2016) and is the holy grail of neurorehabilitation (Brock et al. 2011; Levin and Panturin 2011; Ting et al. 2015). Post-stroke rehabilitation is a complex process requiring neurorehabilitation therapists to integrate their theoretical knowledge of basic science and motor control with professional practice knowledge (Kleim 2011).

This paper will critically discuss the importance of integrating current neuroscience and motor control knowledge in order to better understand and describe the clinical presentation of persons post-stroke such that the effectiveness of stroke rehabilitation can be appropriately measured. In fact, the need to translate this knowledge when evaluating motor recovery, specifically with respect to measuring movement performance, has been identified as crucial in determining the effectiveness of neurorehabilitation interventions (Jolkkonen and Kwakkel 2016; Stinear 2016).

\section{Post-stroke movement deficits - understanding the contribution of the cortico-reticular pathways in movement control}

Identifying and understanding the impact of a focal cerebral lesion on a person's movement capability from a 
neurophysiological perspective, specifically with respect to fibre tract involvement, remains a considerable challenge within the stroke neurorehabilitation setting (Grefkes and Ward 2014). Explicating the neurophysiological implications of a cerebral lesion with respect to the clinical presentation is extremely complex. This is made even more difficult due to our evolving understanding that even localized lesions will influence remote linked neural areas due to the process of secondary denervation known as diaschisis (Silasi and Murphy 2014). In fact, both cortico-spinal and reticulospinal pathways maybe affected, with contra-lesional motor impairments primarily due to cortico-spinal involvement, as well as ipsi- and contra-lesional postural control impairments due to the involvement of bilateral reticulospinal pathways (Lemon 2008; Sousa, Silva et al. 2012; Silva, Sousa et al. 2017).

Thus, whilst understanding the size and location of the focal lesion is important with respect to the impact on brain networks (Grefkes and Ward 2014), it is also important to consider the primary neural targets of the lesioned area as the impact of secondary denervation will contribute to the individual clinical presentation (Nguyen and Botez 1998; Carter et al. 2012; Fornito et al. 2015). Based on the understanding of the nature and role of the cortico-reticulospinal pathways, careful consideration should be addressed not only to the contralesional deficits, but also to the bilateral postural control deficits (Silva, Sousa et al. 2013; Silva, Silva et al. 2014), and the need for sensitive measures to evaluate this important aspect of post-stroke movement dysfunction. Similarly, evidence suggests that the cortico-reticular system also has a role in more distal motor control (Riddle et al. 2009; Soteropoulos et al. 2012; Honeycutt et al. 2013). Recent evidence suggests that spinal cord interneurons involved in hand movements receive convergent input from both reticulospinal and cortico-spinal systems (Riddle and Baker 2010). Additionally, ipsilesional lower limb impairments due to reticulospinal involvement have also been identified in persons post-stroke (Sousa, Silva et al. 2013; Silva, Sousa et al. 2015). Therefore, our programme of research investigating the ipsi and contralesional deficits in persons post-stroke (Silva, Sousa et al. 2013; Sousa, Silva et al. 2013, Silva, Silva et al. 2014; Silva, Sousa et al. 2015,; Sousa, Silva et al. 2015, Sousa, Silva et al. 2015), along with increasing neuroscientific knowledge with respect to the bilateral nature of the cortico-reticulospinal system and its extensive contribution to motor control, highlight this issue (Riddle et al. 2009; Jang, Chang et al. 2013; Jang and Lee 2016). This provides a knowledge base suggesting that a person post-stoke is highly likely to have both ipsi and contralesional deficits and, as such, the ipsi-lesional side can no longer be considered the un-lesioned side. (Arya and Pandian 2014; Cavallari et al. 2016; Dietz and Schrafl-Altermatt 2016).

\section{The role of postural control in movement recovery post-stroke}

Postural control from the clinician's perspective is considered an integral aspect of task performance and movement quality (Vaughan-Graham and Cott 2016) and has been discussed by numerous scholars (Bernstein 1967; Winter et al. 1990; Aruin 2003; Feldman and Levin 2009).

Although there is no consensus on one motor control theory (Levin 2016), there is strong evidence for the role of anticipatory postural control as an integral aspect of movement control (Takakusaki 2013) revealing once more the important role of the cortico-reticulospinal system in motor control and movement recovery post-stroke (Zaaimi et al. 2012). In fact, the cortico-reticulospinal system is considered one of the major descending pathways by which the brain controls spinal motor output in all vertebrates (Lemon, Landau et al. 2012; Soteropoulos et al. 2012). The importance of the role of the cortico-reticulospinal system in humans has already been highlighted due to some of the resulting motor impairments identified in persons post-stroke (Riddle et al. 2009).

Postural control has recently been described as one of the primary dysfunctions due to neurological conditions (Latash and Huang 2015), including post stroke (Genthon et al. 2008). From a clinical perspective, postural control and movement are considered interactive and interdependent (Leonard et al. 2011; Takakusaki 2013; Vaughan-Graham and Cott 2017) therefore movement problems arising due to stroke may be related to postural control dysfunction. Thus, the evaluation and treatment of postural control should be considered an essential aspect of movement rehabilitation for persons post-stroke in order to minimize the development of compensatory strategies (Jones 2017). Therefore, greater attention to the role of the trunk and both ipsi- and contra-lesional body segments, is required in movement rehabilitation (Levin 1996; Takeuchi and Izumi 2012), rather than sole focus on the role of the upper and lower limb for functional task performance (Cott et al. 2011). The postural control and selective movement components of all tasks, and their relative interaction, are dependent upon in part to integrated bilateral reticular system activity (Honeycutt et al. 2013). Therefore, the consideration of the relative interaction of postural control and selective movement through biomechanical parameters and muscle synergy analysis is required in functional movement analysis as well as in stroke measurement tools (Genthon et al. 2008; Latash and Huang 2015).

\section{Measuring post-stroke deficits and effectiveness of stroke rehabilitation interventions}

Health systems face with considerable pressures due to the increasing prevalence of stroke, particularly in terms of budget constraints, health organization, data and information technology (Truelsen and Bonita 2009; Murray et al. 2012; Krishnamurthi et al. 2013). Consequently, health care systems are required to adapt, developing new solutions and services, affecting the way stroke instruments are used by professionals across the continuum of care (AHA/ACC 2000; Delnoij et al. 2003; Hofmarcher et al. 2007). Furthermore, throughout the care pathway, instruments and scales should contribute to clearly documenting the stroke recovery process across the core multidisciplinary stroke rehabilitation team, as well 
as providing a tool for communication with the person poststroke, the family, the caregiver and within broader societal contexts (NICE 2013). Additionally, it is critical that stroke instruments integrate current neuroscience and motor control knowledge, in order to detect clinically relevant changes throughout the rehabilitation process, and through changes scores contribute to the therapist's clinical reasoning process to optimize stroke rehabilitation outcomes (Beninato and Portney 2011). Therefore, the identification of valid, reliable and clinically relevant outcome measures to document and evaluate post-stroke recovery is vital in determining the effectiveness of interventions (Fulk and Field-Fote 2011).

Historically, primary impairments post-stroke are considered contra-lesional on which stroke instruments measuring change in motor behaviour and function are mainly focused. However, evidence suggests that lesions affecting solely the cortico-spinal system do not strongly interfere with functionality (Lemon, Landau et al. 2012) when compared to lesions affecting network connections between the cerebral cortex and reticular formation (Rothwell 2009).

Although, there is a growing body of knowledge with respect to bilateral postural control impairment in persons post-stroke (Sousa, Silva et al. 2012; Kitsos et al. 2013; Silva, Sousa et al. 2013; Sousa, Silva et al. 2013; Sousa, Silva et al. 2013; Silva, Silva et al. 2014; Silva, Sousa et al. 2015; Sousa, Silva et al. 2015; Sousa, Silva et al. 2015; Silva, Sousa et al. 2017), the current evidence is limited to biomechanical data within the research environment. Despite physiotherapist's observational skills being considered a valuable tool, especially when referring to expert professionals (McGinley et al. 2003), there is a profound lack of objective measures to allow valid and reliable quantification of postural control variables within the clinical setting. Additionally, the majority of motor impairment measures focus on movement-related problems with respect to the contra-lesional side (e.g., Reach Performance Scale, Motor Evaluation Scale for Upper Extremity in Stroke Patients) (Levin, Desrosiers et al. 2004; Johansson and Hager 2012), with little to no consideration of the ipsi-lesional postural control requirements for contralesional movement. In fact, stroke instruments and scales (e.g., Fugl-Meyer Scale), in which the score attribution to the movement performance of the contra-lesional side are based on comparative analysis with the so-called 'non-lesioned/ unaffected/normal side' (Fugl-Meyer et al. 1975; Carr et al. 1985; Duncan et al. 2000), are no longer consistent with the current neuroscientific evidence and may inadvertently limit the assessment, evaluation and clinical reasoning required for neurorehabilitation approaches that consider the function and motor performance of the whole person (VaughanGraham and Cott 2016).

In addition and unfortunately to date, the majority of stroke instruments primarily focus on either the time to complete an activity/task (e.g., $10 \mathrm{~m}$ walk test) (Tilson et al. 2010), the amount of assistance required or the need for an assistive device for task accomplishment (e.g., Functional Independence Measure) (Beninato, Gill-Body et al. 2006), or the level of dependence (e.g., Barthel Index, Balance Berg Scale, Stream Measure) (Mahoney and Barthel 1965; Berg et al. 1995; Daley et al. 1999), rather than individual aspects of functional ability or actual motor performance (VaughanGraham, Cott et al. 2015). This obscures the complex and important role of movement rehabilitation with respect to movement performance, whilst simultaneously limiting the discussion on the aspects of movement recovery that are important to persons post-stroke, clinicians and researchers (Levin, Kleim et al. 2009).

Thus, there is an urgent need for the development of neurorehabilitation outcome measures that reflect current neuroscience and motor control knowledge such that the clinical presentation can be more accurately elucidated and measured over the neurorehabilitation intervention period. Such measures would potentially provide greater insight with respect to the complex clinical presentation whilst, also providing a significant contribution to the knowledge based particularly with respect to prognostic indicators and recovery potential post-stroke.

\section{Future considerations for neurorehabilitation and research}

The developing knowledge based on the role of the corticoreticulospinal system with respect to postural control, specifically the role of feedforward mechanisms such as anticipatory postural adjustments (Takakusaki 2013), support the importance of bilateral postural control for contralateral motor performance (Sousa, Silva et al. 2013; Silva, Silva et al. 2014). The challenge for neurorehabilitation practice and research is quantifying the qualitative aspects of movement in order for effectiveness research to be more clinically relevant (Vaughan-Graham, Cott et al. 2015). Therefore, it is important to consider this issue with respect to stroke instruments and scales in order to optimize analysis, evaluation and treatment of persons with neurological conditions (Latash and Huang 2015). We also agree with Kitsos and colleagues (2013), who suggest that terms such as ipsilesional and contralesional may improve the description of movement dysfunction post central nervous system injury (Kitsos et al. 2013).

The recent suggestion by Latash and Huang (2015), that aspects of movement dysfunction could be considered as impaired control of stability (Latash and Huang 2015) which is consistent with a recent study of expert neurorehabilitation therapists in which postural control (stability), and selective movement (mobility), were integral to their conceptualization of movement (Vaughan-Graham and Cott 2017). Therefore, consideration of the role of postural control/stability as a theory-based clinical descriptor of movement performance is an important issue in neurorehabilitation as well as in the consideration of appropriate and relevant stroke instruments and scales (Latash and Huang 2015).

This paper has discussed the important bilateral role of the cortico-reticulospinal system in motor control and movement recovery following a central nervous system (CNS) lesion. We suggest that the contribution of the ipsilesional cortico-reticulospinal pathways with respect to postural control and subsequent contra-lesional motor performance 
requires consideration in the assessment, evaluation and treatment of persons post CNS lesion. Thus, a reconceptualization of stroke instruments and scales is required such that the objective measurement of both ipsi and contralesional function and dysfunction and the relative influence on movement performance and functional capability can be explored. We believe that this paradigm shift in movement analysis, intervention and evaluation would make a positive contribution to the field of neurorehabilitation practice and research.

\section{Disclosure statement}

No potential conflict of interest was reported by the authors.

\section{References}

AHA/ACC. 2000. Measuring and improving quality of care: a report from the American Heart Association/American College of Cardiology first scientific forum on assessment of healthcare quality in cardiovascular disease and stroke. Stroke. 31:1002-1012.

Arber S. 2012. Motor circuits in action: specification, connectivity, and function. Neuron. 74:975-989.

Aruin AS. 2003. The effect of changes in the body configuration on anticipatory postural adjustments. Motor Control. 7:264-277.

Arya KN, Pandian S. 2014. Interlimb neural coupling: implications for poststroke hemiparesis. Ann Phys Rehabil Med. 57:696-713.

Baker SN. 2011. The primate reticulospinal tract, hand function and functional recovery. J Physiol (Lond). 589:5603-5612.

Barra J, Senot P, Auclair L. 2017. Internal model of gravity influences configural body processing. Cognition. 158:208-214.

Beninato M, Gill-Body KM, Salles S, Stark PC, Black-Schaffer RM, Stein J. 2006. Determination of the minimal clinically important difference in the FIM instrument in patients with stroke. Arch Phys Med Rehabil. 87:32-39.

Beninato M, Portney LG. 2011. Applying concepts of responsiveness to patient management in neurologic physical therapy. J Neurol Phys Ther. 35:75-81.

Berg K, Wood-Dauphinee S, Williams Jl. 1995. The balance scale: reliability assessment with elderly residents and patients with an acute stroke. Scand J Rehabil Med. 27:27-36.

Bernstein N. 1967. The co-ordination and regulation of movements. Pergamon Press.

Bouisset S, Do MC. 2008. Posture, dynamic stability, and voluntary movement. Neurophysiol Clin. 38:345-362.

Brock K, Haase G, Rothacher G, Cotton S. 2011. Does physiotherapy based on the Bobath concept, in conjunction with a task practice, achieve greater improvement in walking ability in people with stroke compared to physiotherapy focused on structured task practice alone?: a pilot randomized controlled trial. Clin Rehabil. 25:903-912.

Carr JH, Shepherd RB, Nordholm L, Lynne D. 1985. Investigation of a new motor assessment scale for stroke patients. Phys Ther. 65:175-180.

Carter AR, Shulman GL, Corbetta M. 2012. Why use a connectivity-based approach to study stroke and recovery of function? Neuroimage. 62:2271-2280.

Cavallari P, Bolzoni F, Bruttini C, Esposti R. 2016. The organization and control of intra-limb anticipatory postural adjustments and their role in movement performance. Front Hum Neurosci. 10:525.

Cott CA, Graham JV, Brunton K. 2011. When will the evidence catch up with clinical practice?. Physiother Can. 63:387-390.

Daley K, Mayo N, Wood-Dauphinee S. 1999. Reliability of scores on the stroke rehabilitation assessment of movement (STREAM) measure. Phys Ther. 79:8-19.

de Niet M, Bussmann JB, Ribbers GM, Stam HJ. 2007. The stroke upperlimb activity monitor: its sensitivity to measure hemiplegic upper-limb activity during daily life. Arch Phys Med Rehabil. 88:1121-1126.
Delnoij DM, Klazinga NS, van der Velden K. 2003. Building integrated health systems in central and eastern Europe: an analysis of WHO and World Bank views and their relevance to health systems in transition. Eur J Public Health. 13:240-245.

Dietz V, Schrafl-Altermatt M. 2016. Control of functional movements in healthy and post-stroke subjects: role of neural interlimb coupling. Clin Neurophysiol. 127:2286-2293.

Dimyan MA, Cohen LG. 2011. Neuroplasticity in the context of motor rehabilitation after stroke. Nat Rev Neurol. 7:76-85.

Duncan PW, Jorgensen HS, Wade DT. 2000. Outcome measures in acute stroke trials: a systematic review and some recommendations to improve practice. Stroke. 31:1429-1438.

Feldman AG, Levin MF. 2009. The equilibrium-point hypothesis-past, present and future. Adv Exp Med Biol. 629:699-726.

Fornito A, Zalesky A, Breakspear M. 2015. The connectomics of brain disorders. Nat Rev Neurosci. 16:159-172.

Fugl-Meyer AR, Jaasko L, Leyman I, Olsson S, Steglind S. 1975. The poststroke hemiplegic patient. 1. a method for evaluation of physical performance. Scand J Rehabil Med. 7:13-31.

Fulk G, Field-Fote EC. 2011. Measures of evidence in evidence-based practice. J Neurol Phys Ther. 35:55-56.

Genthon N, Rougier P, Gissot AS, Froger J, Pelissier J, Perennou D. 2008. Contribution of each lower limb to upright standing in stroke patients. Stroke. 39:1793-1799.

Grefkes C, Nowak DA, Wang LE, Dafotakis M, Eickhoff SB, Fink GR. 2010. Modulating cortical connectivity in stroke patients by rTMS assessed with fMRI and dynamic causal modeling. Neurolmage. 50:233-242.

Grefkes C, Ward NS. 2014. Cortical reorganization after stroke: how much and how functional?. Neuroscientist. 20:56-70.

Guigon E. 2010. Active control of bias for the control of posture and movement. J Neurophysiol. 104:1090-1102.

Hofmarcher MM, Oxley H, Rusticelli E. 2007. Improved health system performance through better care coordination. Paris: OECD

Honeycutt CF, Kharouta M, Perreault EJ. 2013. Evidence for reticulospinal contributions to coordinated finger movements in humans. J Neurophysiol. 110:1476-1483.

Horak FB. 2006. Postural orientation and equilibrium: what do we need to know about neural control of balance to prevent falls? Age Ageing. 35 Suppl 2:ii7-ii11.

Jang S, Lee H. 2016. Gait deterioration due to neural degeneration of the corticoreticular pathway: a case report. Neural Regen Res. 11:687-688.

Jang SH, Chang CH, Lee J, Kim CS, Seo JP, Yeo SS. 2013. Functional role of the corticoreticular pathway in chronic stroke patients. Stroke. 44:1099-1104.

Johansson GM, Hager CK. 2012. Measurement properties of the motor evaluation scale for upper extremity in stroke patients (MESUPES). Disabil Rehabil. 34:288-294.

Jolkkonen J, Kwakkel G. 2016. Translational hurdles in stroke recovery studies. Transl Stroke Res. 7:331-342.

Jones TA. 2017. Motor compensation and its effects on neural reorganization after stroke. Nat Rev Neurosci. 18:267-280.

Kitsos GH, Hubbard IJ, Kitsos AR, Parsons MW. 2013. The ipsilesional upper limb can be affected following stroke. Sci World J. 2013:1.

Kleim JA. 2011. Neural plasticity and neurorehabilitation: teaching the new brain old tricks. J Commun Disord. 44:521-528.

Krishnamurthi RV, Feigin VL, Forouzanfar MH, Mensah GA, Connor M, Bennett DA, Moran AE, Sacco RL, Anderson LM, Truelsen T, et al. 2013. Global burden of diseases and G. B. D. S. E. group (2013). Global and regional burden of first-ever ischaemic and haemorrhagic stroke during 1990-2010: findings from the Global burden of disease study 2010. Lancet Glob Health. 1:e259-e281.

Latash ML. 2016. Biomechanics as a window into the neural control of movement. J Hum Kinet. 52:7-20.

Latash ML, Huang X. 2015. Neural control of movement stability: lessons from studies of neurological patients. Neuroscience. 301:39-48.

Latash ML, Zatsiorsky VM. 2016. Biomechanics and motor control. Elsiever

Lemon RN. 2008. Descending pathways in motor control. Annu Rev Neurosci. 31:195-218. 
Lemon RN, Landau W, Tutssel D, Lawrence DG. 2012. Lawrence and Kuypers (1968a, b) revisited: copies of the original filmed material from their classic papers in Brain. Brain. 135:2290-2295.

Leonard JA, Gritsenko V, Ouckama R, Stapley PJ. 2011. Postural adjustments for online corrections of arm movements in standing humans. J Neurophysiol. 105:2375-2388.

Levin MF. 1996. Interjoint coordination during pointing movements is disrupted in spastic hemiparesis. Brain. 119:281-293.

Levin MF. 2016. Principles of motor recovery after neurological injury based on a motor control theory. Adv Exp Med Biol. 957:121-140.

Levin MF, Desrosiers J, Beauchemin D, Bergeron N, Rochette A. 2004. Development and validation of a scale for rating motor compensations used for reaching in patients with hemiparesis: the reaching performance scale. Phys Ther. 84:8-22.

Levin MF, Kleim JA, Wolf SL. 2009. What do motor "recovery" and "compensation" mean in patients following stroke?. Neurorehabil Neural Repair. 23:313-319.

Levin MF, Panturin E. 2011. Sensorimotor integration for functional recovery and the Bobath approach. Motor Control. 15:285-301.

Mahoney Fl, Barthel DW. 1965. Functional evaluation: the Barthel index. Md State Med J. 14:61-65.

McGinley JL, Goldie PA, Greenwood KM, Olney SJ. 2003. Accuracy and reliability of observational gait analysis data: judgments of push-off in gait after stroke. Phys Ther. 83:146-160.

Mohr JP, Grotta JC, Wolf PA, Moskowitz MA, Mayberg MR, Kummer R. 2011. Middle cerebral artery disease. Stroke: pathophysiology, diagnosis, and management. Philadelphia, USA: Elsevier Sounders

Murray CJ, Vos T, Lozano R, Naghavi M, Flaxman AD, Michaud C, Ezzati M, Shibuya K, Salomon JA, Abdalla S, et al. 2012. Disability-adjusted life years (DALYs) for 291 diseases and injuries in 21 regions, 19902010: a systematic analysis for the global burden of disease study 2010. Lancet. 380:2197-2223.

Nguyen DK, Botez MI. 1998. Diaschisis and neurobehavior. Can J Neurol Sci. 25:5-12.

NICE. 2013. Stroke rehabilitation long term rehabilitation after stroke. Clinical guideline. Methods, evidence and recommendations. London, UK: National Institute for Health and Care Excellence

Prentice SD, Drew T. 2001. Contributions of the reticulospinal system to the postural adjustments occurring during voluntary gait modifications. J Neurophysiol. 85:679-698.

Riddle CN, Baker SN. 2010. Convergence of pyramidal and medial brain stem descending pathways onto macaque cervical spinal interneurons. J Neurophysiol. 103:2821-2832.

Riddle CN, Edgley SA, Baker SN. 2009. Direct and indirect connections with upper limb motoneurons from the primate reticulospinal tract. J Neurosci. 29:4993-4999.

Rothwell J. 2009. Meet the brain neurophysiology. Int Rev Neurobiol. 86:51-65.

Rothwell JC. 2012. Overview of neurophysiology of movement control. Clin Neurol Neurosurg. 114:432-435.

Silasi G, Murphy TH. 2014. Stroke and the connectome: how connectivity guides therapeutic intervention. Neuron. 83:1354-1368.

Silva A, Sousa AS, Pinheiro R, Ferraz J, Tavares JM, Santos R, Sousa F. 2013. Activation timing of soleus and tibialis anterior muscles during sit-to-stand and stand-to-sit in post-stroke vs. healthy subjects. Somatosens Mot Res. 30:48-55.

Silva A, Sousa AS, Silva C, Tavares JM, Santos R, Sousa F. 2015. Ankle antagonist coactivation in the double-support phase of walking: stroke vs. healthy subjects. Somatosens Mot Res. 32:153-157.
Silva A, Sousa ASP, Silva CC, Santos R, Tavares JMRS, Sousa F. 2017. The role of the ipsilesional side in the rehabilitation of post-stroke subjects. Somatosens Mot Res. 34:185-188.

Silva CC, Silva A, Sousa A, Pinheiro AR, Bourlinova C, Silva A, Salazar A, Borges C, Crasto C, Correia MV, et al. 2014. Co-activation of upper limb muscles during reaching in post-stroke subjects: an analysis of the contralesional and ipsilesional limbs. J Electromyogr Kinesiol. 24:731-738

Soteropoulos DS, Williams ER, Baker SN. 2012. Cells in the monkey ponto-medullary reticular formation modulate their activity with slow finger movements. J Physiol. 590:4011-4027.

Sousa AS, Silva A, Santos R. 2015. Ankle anticipatory postural adjustments during gait initiation in healthy and post-stroke subjects. Clin Biomech (Bristol, Avon). 30:960-965.

Sousa AS, Silva A, Santos R. 2015. Reliability of two methods for identifying the postural phase of gait initiation in healthy and poststroke subjects. J Appl Biomech. 31:349-356.

Sousa AS, Silva A, Santos R, Sousa F, Tavares JM. 2013. Interlimb coordination during the stance phase of gait in subjects with stroke. Arch Phys Med Rehabil. 94:2515-2522.

Sousa AS, Silva A, Tavares JM. 2012. Biomechanical and neurophysiological mechanisms related to postural control and efficiency of movement: a review. Somatosens Mot Res. 29:131-143.

Sousa AS, Silva A, Tavares JM. 2013. Interlimb relation during the double support phase of gait: an electromyographic, mechanical and energybased analysis. Proc Inst Mech Eng H. 227:327-333.

Stinear CM. 2016. Stroke rehabilitation research needs to be different to make a difference. F1000Res. 5:1467.

Takakusaki K. 2013. Neurophysiology of gait: from the spinal cord to the frontal lobe. Mov Disord. 28:1483-1491.

Takakusaki K, Chiba R, Nozu T, Okumura T. 2016. Brainstem control of locomotion and muscle tone with special reference to the role of the mesopontine tegmentum and medullary reticulospinal systems. J Neural Transm. 123:695-729.

Takeuchi N, Izumi S. 2012. Maladaptive plasticity for motor recovery after stroke: mechanisms and approaches. Neural Plast. 2012:1.

Tilson JK, Sullivan KJ, Cen SY, Rose DK, Koradia CH, Azen SP, Duncan PW, Locomotor Experience Applied Stroke Investigative T. 2010. Meaningful gait speed improvement during the first 60 days poststroke: minimal clinically important difference. Phys Ther. 90:196-208.

Ting LH, Chiel HJ, Trumbower RD, Allen JL, McKay JL, Hackney ME, Kesar TM. 2015. Neuromechanical principles underlying movement modularity and their implications for rehabilitation. Neuron. 86:38-54.

Truelsen T, Bonita R. 2009. The worldwide burden of stroke: current status and future projections. Handb Clin Neurol. 92:327-336.

Vaughan-Graham J, Cott C. 2016. Defining a Bobath clinical framework a modified e-Delphi study. Physiother Theory Pract. 32:612-627.

Vaughan-Graham J, Cott C. 2017. Phronesis: practical wisdom the role of professional practice knowledge in the clinical reasoning of Bobath instructors. J Eval Clin Pract. 23:935-948.

Vaughan-Graham J, Cott C, Wright FV. 2015. The Bobath (NDT) concept in adult neurological rehabilitation: what is the state of the knowledge? A scoping review. Part II: intervention studies perspectives. Disabil Rehabil. 37:1909-1928.

Winter DA, Patla AE, Frank JS. 1990. Assessment of balance control in humans. Med Prog Technol. 16:31-51.

Zaaimi B, Edgley SA, Soteropoulos DS, Baker SN. 2012. Changes in descending motor pathway connectivity after corticospinal tract lesion in macaque monkey. Brain. 135:2277-2289. 